Mathematical Programming 8 (1975) 161-188.

North-Holland Publishing Company

\title{
POLYHEDRAL ANNEXATION IN MIXED INTEGER AND COMBINATORIAL PROGRAMMING
}

\author{
Fred GLOVER \\ University of Colorado, Boulder, U.S.A.
}

Received 24 September 1973

Revised manuscript received 10 January 1975

\begin{abstract}
Polyhedral annexation is a new approach for generating all valid inequalities in mixed integer and combinatorial programming. These include the facets of the convex hull of feasible integer solutions. The approach is capable of exploiting the characteristics of the feasible solution space in regions both "adjacent to" and "distant from" the linear programming vertex without resorting to specialized notions of group theory, convex analysis or projective geometry. The approach also provides new ways for exploiting the "branching inequalities" of branch and bound.
\end{abstract}

\section{Introduction}

This paper provides a new approach, called polyhedral annexation, for generating valid inequalities for mixed integer programming. Polyhedral annexation provides a particularly effective tool for exploiting problem structure. The inequalities it generates take advantage of structure in a completely general way, without requiring any specific form for the constraints defining the feasible (continuous) region. As in the group theoretic approaches, the inequalities are capable of penetrating regions that may be "distant" from the unit hypercube containing the linear programming vertex. At the same time, as in the convex analysis ("outer polar" and "polaroid") approaches, the inequalities profit from local information about the form of the feasible solution space. The inequalities also, however, make fruitful use of relevant constraints that do not affect the local vicinity of the linear programming vertex.

Successively iterated, polyhedral annexation can provide all relevant inequalities for mixed integer programming. These include the inequalities that succeed in converting the mixed integer problem into a linear program. However, the precise range of inequalities that can potentially be generated is less important than the ability to generate inequal- 
ities with particular desired properties. Accordingly, results are given for improving a polyhedral cut by a process of sequential implementation. We also show how to obtain "optimal" inequalities - i.e., supports and facets of the convex hull of feasible solutions - by linear programming. The linear programming problem is expressed in a "primal feasible" form that yields a valid inequality at each iteration. New information is generated as it is needed, proceeding through successive improvements until an optimum or a desired stopping point is reached.

Polyhedral annexation is also a useful supplement to branch and bound. The approach provides new branching schemes as well as improved bounds.

On the negative side of the ledger, polyhedral annexation shares a limitation in common with a number of other efforts to take fuller advantage of problem structure - i.e., its strongest forms require ready access to a substantial amount of information from the updated LP tableau. This is extremely inconvenient for the "product form of the inverse" codes often used in present commercial applications. However, the adaptive aspect of the approach makes it possible to respond to trade-offs between the cost of obtaining updated tableau information and the improvement in the resulting inequality.

\section{Formulation}

The mixed integer programming (MIP) problem will be written $\operatorname{maximize} x_{0}=c x$, subject to $A x=b$,

$$
x_{i} \geqslant 0, \quad i \in M=\{1, \ldots, m\}, x_{i} \text { integer, } i \in I .
$$

The index sets $M$ and $I$ may or may not be disjoint, and may or may not contain the indexes of all components of the vector $x$. We will represent the updated linear programming (LP) tableau format for this problem as

$$
\begin{aligned}
\operatorname{maximize} x_{0} & =x_{00}+\sum_{j \in N} x_{0 j}\left(-t_{j}\right), \\
x_{i} & =x_{i 0}+\sum_{j \in N} x_{i j}\left(-t_{j}\right) \text { for all components } x_{i} \text { of } x,
\end{aligned}
$$


where the $t_{j}$ are the current nonbasic variables and may be assumed to consist of a subset of the $x_{i}, i \in M$. Some of the integer variables $x_{i}, i \in I$, may represent integer combinations and translations of others. Generally, too, we will suppose that each $x_{i}, i \in I$, has a finite range of admissible values, though this assumption is not required to obtain valid inequalities. This foregoing notation is standard except that we have used the same symbol to denote both variables and coefficients (variables being single-subscripted and coefficients double-subscripted). We will follow this convention with respect to the updated tableau (basis) representation of other variables $u_{i}$ and $v_{i}$ subsequently to be introduced; i.e., the current LP representation for these variables will be respectively

$$
u_{i}=u_{i 0}+\sum_{j \in N} u_{i j}\left(-t_{j}\right), \quad v_{i}=v_{i 0}+\sum_{j \in N} v_{i j}\left(-t_{j}\right)
$$

\section{Inequalities from convex domains}

The fundamental ideas for generating valid inequalities for the MIP problem by reference to convex domains have by now received a fairly broad exposure in the literature, and we will review them only briefly.

The terminology for this approach (shared with linear programming) defines the cone corresponding to the LP basis representation to be the set of points generated by allowing the nonbasic variables $t_{j}$ to vary nonnegatively. The $k^{\text {th }}$ edge of the cone is correspondingly the set of points generated by allowing $t_{k}$ to vary nonnegatively, holding all other nonbasic variables constant at 0 .

The vertex of the LP cone is the point obtained by setting all $t_{j}$, $j \in N$, equal to 0 . No assumption is required that this point be optimal or feasible for the LP problem.

The standard result for obtaining cutting planes in this framework can be expressed informally by saying that a valid inequality for the MIP problem arises in the following manner: identify a convex set that contains the LP vertex but no feasible MIP solutions in its interior; extend each edge (typically "as far as possible") so that its (extended) endpoint remains in the convex set; pass a hyperplane through these endpoints; specify the cut to be the associated half space that excludes the LP vertex. In particular, if the extended endpoints are given by the values $t_{j}^{*}, j \in N$, then the cut inequality is 


$$
\sum_{j \in N}\left(1 / t_{j}^{*}\right) t_{j} \geqslant 1
$$

The foregoing approach has its origins in the work of Balas [1], Young [26] and Tui [25]. Early extensions and variations are also given in [10]. Building on these foundations, elegant results have been developed by Balas [2] and Burdet [7] using (extended) tools of convex analysis and projective geometry. An alternative line of development $[11,13,14,15]$, provides the antecedents to the work presented here. This type of approach has concentrated on the identification of polyhedral convex sets ${ }^{1}$ that give improved inequalities for problems with "structure", such as the "multiple choice" problems [11] and the "disjunctive facet" problems [14]. Contemporaneous investigations into this area have been conducted by Balas [3, 4] and Jeroslow [20, 21]. Investigations into related areas have also been conducted by Burdet [7, 8] and Johnson [22, 23].

Polyhedral convex sets have several attractive characteristics. First, the use of a polyhedron to determine (1) enables an edge to be extended in the negative direction, provided the edge strictly recedes from all hyperplanes of the polyhedron when extended in the positive direction ${ }^{2}$. Negative edge extensions yield more powerful cuts, and therefore are desirable when available.

Another attractive feature of polyhedral convex sets is the ease with which the edge extensions giving the cut (1) can be determined. In particular, suppose the polyhedron is given by

$$
v_{p} \geqslant 0, \quad p \in P \text {. }
$$

The value of $v_{p}$ for any given value of $t_{j}$ on the $j^{\text {th }}$ edge of the LP cone is $v_{p 0}-v_{p j} t_{j}$, where the current LP basis representation of $v_{p}$ is $v_{p}=v_{p 0}+\Sigma_{j \in N} v_{p j}\left(-t_{j}\right)$. The condition that the vertex of the LP cone lies in the interior of this polyhedron translates into $v_{p 0}>0, p \in P$. Thereupon, the requirement that the endpoint of the $j^{\text {th }}$ edge lies in (P) for $t_{j}=t_{j}^{*}$ may be expressed as $v_{p 0}-v_{p j} t_{j}^{*} \geqslant 0, p \in P$. The largest value of $t_{j}^{*}$ satisfying this requirement is given by

1 We will use the terms "polyhedral convex set" and "polyhedron" interchangeably - i.e., polyhedron may refer to an unbounded as well as a bounded region. (Sometimes polytope is given this usage.)

2 The first use of negative edge extensions is due to Owen [24]. Related ideas are developed in $\{3,4,12,20]$. 


$$
t_{j}^{*}=\min _{p \in P_{j}^{+}}\left\{v_{p 0} / v_{p j}\right\},
$$

where

$$
P_{j}^{+}=\left\{p \in P: \quad v_{p j} \geqslant 0\right\}
$$

(letting $t_{j}^{*}=\infty$ if $v_{p j}=0$ for all $p \in P$, giving $1 / t_{j}^{*}=0$ ).

On the other hand, if $P_{j}^{+}=\emptyset$, then a negative edge extension is permissible, and $t_{j}^{*}$ may be given by

$$
t_{j}^{*}=\min _{p \in P_{j}^{-}}\left\{v_{p 0} / v_{p j}\right\},
$$

where

$$
P_{j^{*}}=\left\{p \in P: \quad v_{p j}<0\right\} .
$$

As noted in [14], a negative edge extension goes outside the polyhedron (P) whenever more than one hyperplane $v_{p}=0$ is encountered in the negative direction (unless the last and first hyperplane are intersected simultaneously). Also, while negative edge extensions give deeper cuts, the deepest cuts are obtained for the "shallowest" negative extensions - in exact contrast to the situation for positive extensions. Consequently, the precise "shape" and "composition" of the polyhedron (P) importantly affect the strength of the inequalities derived from it.

The goal of this paper is to provide a new framework for dealing with such considerations, and for exploiting the ideas underlying the use of polyhedral convex sets generally.

\section{Fundamental results}

The polyhedral annexation approach applies to a variety of nonconvex mathematical programming problems in addition to the mixed integer programming problem. To make this connection easily accessible, some of the results are stated in slightly more general form than necessary for the MIP problem. (Fortunately, this does not diminish their simplicity or require more difficult proofs.) For results that apply as readily to other problems, such as the disjunctive facet problem, we omit explicit reference to the MIP problem.

Feasibility conditions for the class of problems we consider can be expressed in terms of "building block" polyhedra represented by the system 


$$
\begin{gathered}
v_{h} \geqslant 0, \quad h \in P_{1}, \\
v_{h} \geqslant 0, \quad h \in P_{2}, \\
\vdots \\
v_{h} \geqslant 0, \quad h \in P_{w} .
\end{gathered}
$$

The feasible set $F$ is defined relative to (2) as the set of points that do not lie in the interior of any of the polyhedra of the system. Thus, specifically, a point belongs to $F$ if and only if it satisfies $v_{h} \leqslant 0$ for at least one $h \in P_{1}$ and for at least one $h \in P_{2} \ldots$ and for at least one $h \in P_{w}$. This is logically equivalent to saying $v_{h} \leqslant 0$ for all $h \in H_{1}$, or for all $h \in H_{2} \ldots$ or for all $h \in H_{r}$, where each set $H_{p}, p=1, \ldots, r$, is created by selecting exactly one index $h$ from each set $P_{i}, i=1, \ldots, w$. (Each set $H_{p}$ thus has $w$ elements, except for duplicated indexes, and $r$ is the total number of distinct sets of this form.)

This way of viewing feasibility conditions for combinatorial problems is a common thread of $[11,13,14]$. The first characterization of $F$ may be called a "conjunctive" characterization, the second a "disjunctive" characterization.

In the context of the MIP problem, the system (2) that defines feasibility is

$$
\begin{aligned}
-x_{1} & \geqslant 0, \\
-x_{2} & \geqslant 0, \\
& \vdots \\
-x_{m} & \geqslant 0, \\
u_{1} & \geqslant 0, \quad 1-u_{1} \geqslant 0, \\
u_{2} & \geqslant 0, \quad 1-u_{2} \geqslant 0, \\
& \vdots \\
u_{s} & \geqslant 0, \quad 1-u_{s} \geqslant 0,
\end{aligned}
$$

where the polyhedra $0 \leqslant u_{t} \leqslant 1, t=1, \ldots, s$ correspond to the regions of the form $x_{i}^{*} \leqslant x_{i} \leqslant x_{i}^{*}+1, i \in I$, and where the constants $x_{i}^{*}$ range over admissible values of the integer variables $x_{i}, i \in I$.

Thus, a point is feasible for the MIP problem (by definition) if and 
only if it does not lie in the interior of any of these specified polyhedra (hence satisfies $x_{i} \geqslant 0$ for all $i=1, \ldots, m$, and satisfies $u_{t} \leqslant 0$ or $1-u_{t} \leqslant 0$ for all $t=1, \ldots, s$ ).

The polyhedral annexation principle applies to any two polyhedra that contain no points of $F$ in their interiors. Then, by annexing one to the other, a single new polyhedron is created whose interior also contains no point of $F$. Thus, the principle can be successively applied to the building block polyhedra to create other polyhedra that serve to generate inequalities of the form (1).

The manner in which a particular polyhedron (Q) can be annexed to a polyhedron $(\mathrm{P})$ will now be characterized.

\section{Theorem 4.1. Assume that the polyhedra}

$$
\begin{aligned}
& v_{p} \geqslant 0, \quad p \in P, \\
& v_{q} \geqslant 0, \quad q \in Q
\end{aligned}
$$

contain no feasible solutions in their interiors. Then for any $k \in P$, the new polyhedron

$$
\begin{aligned}
& v_{p} \geqslant 0, \quad p \in P-\{k\}, \\
& \lambda_{k q} v_{k}+\lambda_{q} v_{q} \geqslant 0, \quad q \in Q
\end{aligned}
$$

contains no feasible solutions in its interior for all nonnegative values of the parameters $\lambda_{k q}$ and $\lambda_{q}, q \in Q$.

Proof. Suppose on the contrary the polyhedron (R) contains a feasible solution in its interior. Hence, this solution satisfies $v_{p}>0, p \in P-\{k\}$ and $\lambda_{k q} v_{k}+\lambda_{q} v_{q}>0, q \in Q$. From the first of these inequalities it follows that the solution must also satisfy $v_{k} \leqslant 0$, or else it would be in the interior of $(\mathrm{P})$. Consequently, from the second of the inequalities, with all parameters nonnegative, it follows that $v_{q}>0, q \in Q-$ i.e., the solution lies in the interior of $(\mathrm{Q})$. The theorem is established by contradiction. ${ }^{3}$

It may be noted that the hyperplanes $\lambda_{k q} v_{k}+\lambda_{q} v_{q}=0$ arise geometrically by rotating the hyperplanes $v_{k}=0$ and $v_{q}=0$ through their

3 It should be noted by this proof that we are using the term "interior" in a strictly algebraic sense. This avoids special qualifications requiring subsets of parameters to be positive. In particular, if a polyhedron contains a defining half-space $v_{h} \geqslant 0$ such that $v_{h}=0$, then the polyhedron has no interior, since no point satisfies $0>0$. 
intersection - thus the polyhedron ( $R$ ) arises by deforming (Q) using the indicated rotations and then annexing $(\mathrm{Q})$ to $(\mathrm{P})$ (sans the half space used to deform (Q)). ${ }^{4}$

To get a clearer notion of what Theorem 4.1 is saying, let the index sets defining $(\mathrm{P})$ and $(\mathrm{Q})$ be given by

$$
P=\{1,2,3\}, \quad Q=\{4,5\} .
$$

Then, selecting $k=3$ from $P$, the theorem gives (R) with the index set

$$
R=\{1,2,3-4,3-5\}
$$

where by $3-4$ and $3-5$, we mean that the associated half spaces are obtained from nonnegative linear combinations involving $v_{3}$ and $v_{4}$, and $v_{3}$ and $v_{5}$. Suppose we now take $(\mathrm{R})$ in the role of $(\mathrm{P})$ and repeat the process, choosing $k=2$. Then we obtain the new (R) characterized by the index set

$$
R=\{1,2-4,2-5,3-4,3-5\} .
$$

That is, the theorem says there is no feasible solution in the interior of this latter ( $\mathrm{R})$ for all nonnegative linear combinations involving $v_{2}$ and $v_{4}, v_{2}$ and $v_{5}$, etc. Repeating this process one more time and selecting $k=1$, the final $(\mathrm{R})$ consists of all "paired" halfspaces, where each one is a nonnegative linear combination of exactly one half space from (P) and exactly one half space from (Q).

It is particularly interesting to trace the foregoing process from a logical standpoint. Suppose we use the "index set" notation to summarize the logical relations governing the assumption that $(\mathrm{P})$ and $(\mathrm{Q})$ have no feasible solutions in their interiors. That is, we let $\{1,2,3\}$ represent the conditions $v_{1} \leqslant 0$ or $v_{2} \leqslant 0$ or $v_{3} \leqslant 0$, and let $\{4,5\}$ represent the conditions $v_{4} \leqslant 0$ or $v_{5} \leqslant 0$. Then the "index set" $\{1,2,3-4,3-5\}$ may reasonably be taken to represent $v_{1} \leqslant 0$ or $v_{2} \leqslant 0$ or $\left(v_{3}\right.$ and $\left.v_{4} \leqslant 0\right)$ or $\left(v_{3}\right.$ and $\left.v_{5} \leqslant 0\right)$. But this last collection of disjunctions is a logical consequence of the assumption $v_{1} \leqslant 0$ or $v_{2} \leqslant 0$ or $v_{3} \leqslant 0$ and $v_{4} \leqslant 0$ or $v_{5} \leqslant 0$. Thus, the polyhedral annexation principle may be viewed as a means for generating polyhedra, and hence inequalities, that "correspond" to logical consequences of the original feasibility assumptions. ${ }^{5}$ As will be seen, this "correspondence" is complete, in

4 In view of this, the annexation process may be conceived as more nearly "political" than "territorial", i.e., the annexed body may undergo a radical transformation in the process of being assimilated into the main body.

5 I am indebted to Bob Jeroslow for pointing out this interpretation to me. 
the sense that it gives rise to inequalities that characterize the convex hull of $F$.

In order to consider successive applications of Theorem 1, as in the preceding illustration, we will generally assume that the hyperplanes $v_{p}=0$ of the polyhedron $(\mathrm{P})$ are themselves obtained as sequences of nonnegative rotations of other hyperplanes $v_{h}=0$, i.e., that $(\mathrm{P})$ may be written

$\left(\mathrm{P}_{\lambda}\right) \quad v_{p}=\sum_{h \in H_{p}} \lambda_{p h} v_{h} \geqslant 0, \quad p \in P$,

where we assume that $\left(\mathrm{P}_{\lambda}\right)$ has no feasible points in its interior for all nonnegative values of the parameters $\lambda_{p h}$.

For example, repeated application of Theorem 1 to the polyhedra of (2) yields a form of $\left(\mathrm{P}_{\lambda}\right)$ in which each set $H_{p}$ contains exactly one index from each set $P_{1}, \ldots, P_{w}$ and the collection $H_{p}, p \in P$, consists of all such sets. (The construction proceeds exactly as in the earlier illustration, taking each new (R) in the role of (P) and allowing $Q$ to change from $P_{t}$ to $P_{t+1}$ as soon as the elements of $P_{t}$ have been distributed across each component of the current "index set" for $(\mathrm{R}).)^{6}$ The resulting sets $H_{p}, p \in P$, are the same as those that provide the "disjunctive" characterization of the feasible set $F$. To distinguish these particular sets $I_{p}$ from others that can be obtained by Theorem 4.1, we denote these sets by $I_{p}^{*}, p \in P^{*}$, and denote the corresponding polyhedron $\left(\mathrm{P}_{\lambda}\right)$ by $\left(\mathrm{P}_{\lambda}^{*}\right)$.

In particular, then, we have observed that the polyhedral annexation principle implies that $\left(\mathrm{P}_{\lambda}^{*}\right)$ contains no points of $F$ in its interior, for all nonnegative parameter values, where the sets $H_{p}^{*}, p \in P^{*}$ correspond to the elements of the cross product set $P_{1} \times P_{2} \times \ldots \times P_{w}$, and where the feasible set $F$ is the set of points satisfying $v_{h} \leqslant 0$ for all $h \in H_{p}^{*}$ and some $p \in P$.

We can now state the following results.

Theorem 4.2. If $v_{0} \leqslant 0$ is any inequality satisfied by all points in $F$, then the half space $v_{0} \geqslant 0$ can be obtained by successive application of Theorem 4.1 to the polyhedra of (2).

Proof. Given any $p \in P^{*}$, if $v_{h} \leqslant 0$ for all $h \in I_{p}^{*}$, then by the disjunctive characterization of $F$ it follows that $v_{0} \leqslant 0$. Consequently, there exist nonnegative numbers $\lambda_{p h}^{*}$ such that

6 This construction treats the elements of each set $P_{t}$ as different from those of all other sets, and therefore may contain "redundancy" if the index sets of (2) are not pairwise disjoint. 


$$
\sum_{h \in H_{p}^{*}} \lambda_{p h}^{*} v_{h}=v_{0}, \quad p \in P^{*}
$$

Assigning the parameters of the polyhedron $\left(\mathrm{P}_{\lambda}^{*}\right)$ the values $\lambda_{p h}=\lambda_{p h}^{*}$ thus reduces every half space defining this polyhedron to the form $v_{0} \geqslant 0$. This completes the proof.

The principal consequence of this theorem is the following.

Corollary 4.3. Assume $v_{0}=0$ is a support (or facet) of the convex hull $F^{*}$ of $F$ such that the inequality $v_{0} \leqslant 0$ is not satisfied by the current $L P$ basic solution. Then $v_{0} \leqslant 0$ can be obtained as a cut inequality (1) by successive application of polyhedral annexation.

Proof. The proof is immediate by virtue of Theorem 4.2, since the LP vertex lies in the interior of $v_{0} \geqslant 0$, and hence by the rule for obtaining (1) it follows that the cut inequality yields $v_{0} \leqslant 0$ from the polyhedral half space $v_{0} \geqslant 0$.

(Note that if $F$ is empty, we may take the inequality $v_{0} \leqslant 0$ of Theorem 4.2 and Corollary 4.3 to be $1 \leqslant 0$. Then, Theorem 4.2 yields the polyhedron $1 \geqslant 0$. The inequality (1) for this polyhedron has the form $0 \geqslant 1$, corresponding to $v_{0} \leqslant 0$.)

It is of course entirely possible (and likely) that many supports and facets of $F^{*}$ can be obtained without recourse to the system $\left(\mathrm{P}_{\lambda}^{*}\right)$, which can be excessively large. The ability to generate polyhedra $\left(\mathbf{P}_{\lambda}\right)$ at stages "intermediate" to $\left(\mathrm{P}_{\lambda}^{*}\right)$ is extremely important in this regard. Results that permit this to be done effectively constitute the main focus of the remainder of this paper.

Before proceeding to more advanced considerations, a few preliminary observations can be made concerning the range and structure of the polyhedra $\left(\mathrm{P}_{\lambda}\right)$ that produce inequalities as a consequence of the polyhedral annexation principle. That is, viewing Theorem 4.1 as an inductive tool, it is of interest to know the type of polyhedra it validates in the context of (1), and to take note of simple rules by which these polyhedra arise. In fact, it is entirely sufficient from an inductive standpoint to restrict attention to constructions in which (Q) is one of the building block polyhedra.

As in the construction of the earlier illustration, and in the construction that yields $\left(\mathrm{P}_{\lambda}^{*}\right)$, we treat each new $(\mathrm{R})$ in the role of $(\mathrm{P})$. There is 
one obviously nonproductive application of Theorem 4.1, but otherwise any sequence of steps produces an (R) at each iteration which contains "more information" than the $(\mathrm{P})$ from which it was derived. The nonproductive application results when the half spaces defining (R) include the half spaces defining (P). To illustrate, suppose $P=\{1,2,3-4$, $3-5\}$ and $Q=\{5,6\}$, where $P$ and $Q$ are expressed in terms of the index set notation introduced earlier.

If the half space associated with " $3-5$ " of $P$ is assigned the role of $v_{k} \geqslant 0$ in Theorem 4.1, the resulting index set for $(\mathrm{R})$ is

$$
R=\{1,2,3-4,3-5-5,3-5-6\} .
$$

But " $3-5-5$ " is the same as " $3-5$ " (since parameterizing $v_{5} \geqslant 0$ twice accomplishes nothing beyond parameterizing it once), and hence $R$ "includes" $P$. This means that any cut obtained from (R) can already be obtained from $(\mathrm{P})$, and hence there is no point in carrying out the step to obtain ( R). (The rule to avoid this type of step when $(\mathrm{Q})$ is a building block polyhedron is particularly simple: exclude any step for which the set $H_{k}$ associated with $v_{k}$ contains an index of $Q$. Note that "3-5" identifies $H_{k}$ in the example just cited.)

It is easy to show that every construction that iteratively replaces $(\mathrm{P})$ by $(R)$ and takes $(Q)$ to be a building block polyhedron will ultimately yield $\left(\mathrm{P}_{\lambda}^{*}\right)$, provided only that nonproductive steps are excluded. (This holds no matter which polyhedron is initially taken to be (P), as long as it has no feasible solutions in its interior, and allowing for "redundancy" in the characterization of $\left(\mathrm{P}_{\lambda}^{*}\right)$.) In fact, if the initial (P) is a building block polyhedron, and if no $P_{t}$ is a subset of any other (in which case the larger $P_{t}$ could always be dropped) then this construction yields all polyhedra $\left(\mathrm{P}_{\lambda}\right)$ whose sets $H_{p}$ can be "ordered" in the following manner:

(i) All "first indexes" of the sets $H_{p}, p \in P$, constitute the elements of a single $P_{t}$ (i.e., all elements of this $P_{t}$ are included, some possibly more than once).

(ii) For any collection of sets $H_{p}$ that have a common first index, all second indexes (if any exist) constitute the elements of a single $P_{t}$ (different from the $P_{t}$ of (i)).

(iii) For any collection of sets $H_{p}$ that have common first and second indexes, all third indexes (if any exist) constitute the elements of a single particular $P_{t}$ (different from the $P_{t}$ of (i) and (ii)) - and so forth for subsequent indexes.

(iv) No ordered set $H_{p}$ is the same as any other, and if the sets $P_{t}$ are 
pairwise disjoint (as by assigning an element a different index for each set in which it appears), then no $H_{p}$ is the same as any other, regardless of ordering.

These conditions can be verified simply by taking "first indexes" to correspond to the elements of an initial $P$, and taking the sets $P_{t}$ of (ii), (iii), etc., to correspond to sets $Q$. These $Q$ may be in any sequence as long as a $Q$ associated with a "second index" is introduced before a $Q$ associated with a "third index", etc. (A "third index" $Q$ can be introduced before a "second index" $Q$ provided they are not linked in the manner indicated in (iii).)

Thus, all polyhedra $\left(\mathrm{P}_{\lambda}\right)$ satisfying the conditions (i) - (iv) are validated by Theorem 4.1. Having found a good $\left(\mathrm{P}_{\lambda}\right)$ for a particular class of problems, either empirically or analytically, one can start with this $\left(\mathrm{P}_{\lambda}\right)$ to initiate the solution of other problems of the same class. $A\left(\mathrm{P}_{\lambda}\right)$ which is too large for a particular problem (due to the number and composition of the sets $P_{t}$ for that problem) can be "collapsed" to a simpler form simply by setting selected parameters to 0 . Thus, for instance, given any subset $P^{\prime}$ of $P$, if there is a nonempty subset $H^{\prime}$ of each $H_{p}$, $p \in P^{\prime}$, then the entire collection $H_{p}, p \in P^{\prime}$ can be replaced by $H^{\prime}$ (replacing the associated half spaces by a single half space). This amounts to setting all parameters $\lambda_{p h}=0$ for $p \in P^{\prime}$ and $h \in H^{\prime}$.

While these observations help to convey the range of strategic possibilities that are available, they do little to provide concrete guidelines for applying such strategies intelligently.

To complete the foundation for such guidelines, especially in the context of the MIP problem, we state two direct consequences of Theorem 4.1 under the assumption that $(\mathrm{Q})$ consists only of the building block polyhedra $0 \leqslant u_{t} \leqslant 1$ and $x_{i} \leqslant 0$.

Corollary 4.4. Assume that $(\mathrm{P})$ has the form $\left(\mathrm{P}_{\lambda}\right)$ and has no feasible MIP solutions in its interior for all nonnegative values of the parameters $\lambda_{p h}$. For any chosen index $k \in P$, let

$$
\begin{aligned}
& v_{k}^{\prime}=\lambda_{t}^{\prime} u_{t}+\sum_{h \in H_{k}} \lambda_{k h}^{\prime} v_{h}, \\
& v_{k}^{\prime \prime}=\lambda_{t}^{\prime \prime}\left(1-u_{t}\right)+\sum_{h \in H_{k}} \lambda_{k h}^{\prime \prime} v_{h} .
\end{aligned}
$$

Then the polyhedron 


$$
\begin{aligned}
& v_{p} \geqslant 0, \quad p \in P-\{k\}, \\
& v_{k}^{\prime} \geqslant 0, \quad v_{k}^{\prime \prime} \geqslant 0
\end{aligned}
$$

contains no feasible MIP solutions in its interior for all nonnegative values of the parameters $\lambda_{p h}, \lambda_{k h}^{\prime}, \lambda_{k h}^{\prime \prime}, \lambda_{t}^{\prime}$ and $\lambda_{t}^{\prime \prime} \cdot 7$

We stress that the polyhedron $0 \leqslant u_{t} \leqslant 1$ need not be selected so that the LP vertex lies in its interior in order for the "final polyhedron" of Corollary 4.4 to have this property. The same comment especially applies to the polyhedron $x_{i} \leqslant 0$ implicit in the following corollary (which never contains a feasible LP vertex in its interior).

Corollary 4.5. Assume (P) satisfies the conditions of Corollary 4.4, and for any selected index $k \in P$, and any $i \in M$, let

$$
v_{k}^{\prime}=\lambda_{i}^{\prime}\left(-x_{i}\right)+\sum_{h \in H_{k}} \lambda_{k h} v_{h}
$$

Then the polyhedron

$$
\begin{aligned}
& v_{p} \geqslant 0, \quad p \in P \ldots\{k\}, \\
& v_{k}^{\prime} \geqslant 0
\end{aligned}
$$

contains no feasible MIP solutions in its interior for all nonnegative values of $\lambda_{i}^{\prime}$ and the parameters $\lambda_{p h}, p \in P$.

An important aspect of Corollary 4.5 deserves mention. If the constraint of the MIP problem is not $x_{i} \geqslant 0$ but $x_{i}=0$, then the corollary implies that the parameter $\lambda_{i}^{\prime}$ need not be nonnegative but can be unrestricted. Similarly, in the application of Corollary 4.4 , if one of the variables $u_{t}$ or $1-u_{t}$ corresponds to one of the $x_{i}, i \in M$ (as, for example, in the $0-1$ problem), it follows from Corollary 4.5 that the parameter of this variable can likewise be unrestricted. It is easy to see that the assumptions of Corollaries 4.4 and 4.5 (and of a "parameterized" version of Theorem 4.1) can be modified to replace nonnegativity requirements on parameters of $(P)$ and $(Q)$ by any other requirements,

7 In applications of Corollary 4.4 it is useful to consider integer variables $u_{t}$ and $1-u_{t}$ created by simple integer combinations of the "original" variables to permit deeper edge exiensions relative to the current LP basis. (E.g., one might use the variables created by reducing the coefficients of nonbasic integer variables to their fractional parts.) 
provided these requirements are imposed for corresponding parameters in the new polyhedron. ${ }^{8}$

Another useful aspect of Corollary 4.5 is that it allows the same hyperplane $-x_{i}=0$ (for an arbitrary $i \in M$ ) to rotate any of the hyperplanes of the polyhedron (P) without changing the number of hyperplanes defining $(\mathrm{P})$. Stated in another way, all hyperplanes $-x_{i}=0$, $i \in M$, may be used to (nonnegatively) rotate all hyperplanes $v_{p}=0$, $p \in P$, taking advantage of the full set of constraining inequalities $x_{i} \geqslant 0$, $i \in M$. (These, it should be remembered, consist of all inequalities defining the LP problem, not merely those requiring nonnegativity for "structural" variables.) Thus, the corollary leads to a particularly convenient exploitation of the LP problem structure, without having to specify in advance what that structure may be.

We now turn to the derivation of special relationships that can be used in such applications.

\section{Determining improved polyhedra}

In this section we narrow our focus to polyhedra which (with the exception of the annexed polyhedron (Q)) contain the LP vertex in their interiors, and thereby examine the conditions under which legitimate inequalities are obtained relative to a specific LP basis. Our principal concern in doing this will be to provide demonstrable relationships that can be used in the determination of improved polyhedra by specific annexation strategies.

We will consider two types of strategies: sequential implementation and linear programming. The sequential implementation approach is computationally the least costly (although the amount of effort expended in either approach can be regulated), and most of our results bear directly on conditions affecting its use. The linear programming approach, in contrast, offers the chance to determine "optimal" cuts -

8 A version of Theorem 4.1 that embodies these observations can be stated as follows.

Let $V_{P}$ and $V_{Q}$ be master vectors ("ordered sets") whose components are $v_{p}, p \in P$ and $v_{q}, q \in Q$, and let $Z_{P}$ and $Z_{Q}$ be specific sets of vectors $V_{P}$ and $V_{Q}$. Assume that the polyhedra (P) and (Q) contain no feasible solutions in their interiors for $V_{P} \in Z_{P}$ and $V_{Q} \in Z_{Q}$. Then the polyhedron $(\mathrm{R})$ contains no feasible solutions in its interior for all nonnegative values of $\lambda_{k q}, \lambda_{q}, q \in Q$ and $V_{P} \in Z_{P}, V_{Q} \in Z_{Q}$. Moreover, if $V_{Q} \in Z_{Q}$ implies $v_{q} \geqslant 0$ for all feasible solutions, then the parameter $\lambda_{q}$ need not be nonnegative. Likewise, if $V_{P} \in Z_{P}$ implies $v_{k} \geqslant 0$ for all feasible solutions, then the parameters $\lambda_{k q}$ need not be nonnegative for all $q \in Q$. 
i.e., supports and facets of $F^{*}$ - if the solution of the linear program is carried to completion. This is unnecessary, however, because the calculations can be maintained primal feasible at each stage, and the polyhedron available at any desired stopping point will be currently best. The results for the sequential implementation approach also permit significant computational savings for the linear programming approach by providing "screening" criteria that eliminate certain unprofitable alternatives without having to submit them to the machinery of the simplex method. Of course, intermediate strategies using both sequential implementation and linear programming are possible.

\subsection{Sequential implementation}

To simplify the algebraic expressions and facilitate the proof of the results that follow, we will suppose that the LP basis representations of the variables $v_{p}, p \in P$, have been normalized so that the constant terms $\left(v_{p 0}\right)$ are equal to 1 . This is permissible under the assumption that the polyhedron (2) contains the LP vertex in its interior, since then $v_{p 0}>0$ for all $p \in P$, and the normalization occurs by dividing $v_{p}$ by $v_{p 0}$. We will similarly impose such a normalization on the LP basis representation of $\lambda_{k q} v_{k}+\lambda_{q} v_{q}$ (associated with the polyhedron (R) of Theorem $4.1)$ by requiring $\lambda_{k q} v_{k 0}+\lambda_{q} v_{q 0}=1$, or more simply, $\lambda_{k q}+\lambda_{q} v_{q 0}=1$ (since $k \in P$ and $v_{k 0}=1$ ). This is consistent due to our intention that the polyhedron $(\mathrm{R})$, like $(\mathrm{P})$, contains the LP vertex in its interior. (Since we make no corresponding assumption concerning the annexed polyhedron $(\mathrm{Q})$, we do not attempt to normalize the variables $v_{q}$, $q \in Q$.)

Note that, by the normalization conventions, the polyhedron (R) gives rise to a valid inequality (1) provided only $\lambda_{q} \geqslant 0$ and $\lambda_{q} v_{q 0} \leqslant 1$, $q \in Q$. We will call $\lambda_{q}$ legitimate if it satisfies these conditions.

There are three concepts which, in addition to the normalization conventions, are particularly useful for the following development. The first concerns the "restrictiveness" of the half spaces $v_{k} \geqslant 0$ and $\lambda_{k q} v_{k}+\lambda_{q} v_{q} \geqslant 0$ of Theorem 4.1 relative to a given edge of the LP cone. We will say that $\lambda_{k q} v_{k}+\lambda_{q} v_{q} \geqslant 0$ is less restrictive than $v_{k} \geqslant 0$ relative to edge $j$ if it permits an improved extension of this edge, disregarding all other half spaces that may affect this extension. More formally, if the $j^{\text {th }}$ edge intersects the hyperplanes $v_{k}=0$ and $\lambda_{k q} v_{k}+$ $\lambda_{q} v_{q}=0$ for $t_{j}=t_{j}^{\prime}$ and $t_{j}=t_{j}^{\prime \prime}$, respectively, then $\lambda_{k q} v_{k}+\lambda_{q} v_{q} \geqslant 0$ will be said to be less restrictive than $v_{k} \geqslant 0$ if $1 / t_{j}^{\prime}>1 / t_{j}^{\prime \prime}$ (where reciprocals 
are used to accommodate negative as well as positive edge extensions). This notion is of course pertinent to the question of whether replacing $v_{k} \geqslant 0$ by $\lambda_{k q} v_{k}+\lambda_{q} v_{q} \geqslant 0$ in Theorem 4.2 may yield a better cut via (R) than $(\mathrm{P})$.

The second concept, closely related to that of restrictiveness, is that of a "blocking hyperplane." Specifically, the hyperplane $v_{k}=0$ will be said to block the $j^{\text {th }}$ edge of the LP cone if this hyperplane is intersected by the edge for $t_{j}=t_{j}^{*}$, where $t_{j}^{*}$ is the value of $t_{j}$ for the cut (1). In other words, if $v_{k}=0$ blocks the $j^{\text {th }}$ edge, then the edge extension cannot be improved until $v_{k}=0$ is moved (though the edge may still be blocked by a second hyperplane). The link to the notion of restrictiveness is apparent: there is no point in removing $v_{k}=0$ and replacing it by $\lambda_{k q} v_{k}+\lambda_{q} v_{q}=0$, if the goal is to improve the extension of the $j^{\text {th }}$ edge, unless $\lambda_{k q} v_{k}+\lambda_{q} v_{q} \geqslant 0$ is less restrictive relative to this edge than $v_{k} \geqslant 0$.

The final concept, also closely related to the two preceding, is that of a "barrier" hyperplane. The hyperplane $v_{q}=0$ will be called a barrier for edge $j$ if the hyperplane is approached (or increasingly bypassed) as the edge is extended in a positive direction - or more precisely, if the value of $v_{q}$ decreases as $t_{j}$ increases. (Note that algebraically this simply says that the coefficient $v_{q j}$ is positive in the LP basis representation of $v_{q}$.) A barrier hyperplane for a given edge may be thought of as one that is potentially blocking in the positive direction. A hyperplane that is not a barrier of course poses no obstacle to extending an edge to a greater positive depth. This concept is particularly useful in the application of Corollary 4.4 due to the observation that exactly one of the two hyperplanes $u_{t}=0$ and $1-u_{t}=0$ is a barrier for any given edge (with the exception that neither will be a barrier if they are parallel to the edge).

A useful result concerning the concept of restrictiveness is the following.

Theorem 5.1. Assume $\lambda_{q}$ is positive. Then $\lambda_{k q} v_{k}+\lambda_{q} v_{q} \geqslant 0$ is less restrictive than $v_{k} \geqslant 0$ relative to the $j^{\text {th }}$ edge of the LP cone if and only if the intersection of the $j^{\text {th }}$ edge with $v_{k}=0$ lies in the interior of the half space $v_{q} \geqslant 0$ when the intersecting value of $t_{j}$ is positive, and lies in the interior of the half space $v_{q} \leqslant 0$ when the intersecting value of $t_{j}$ is negative.

Proof. Denote the values of $t_{j}$ at which the $j^{\text {th }}$ edge intersects $v_{k}=0$ 
and $\lambda_{k q} v_{k}+\lambda_{q} v_{q}=0$ by $t_{j}^{\prime}$ and $t_{j}^{\prime \prime}$, respectively, as in the definition of restrictiveness. We must show that $1 / t_{j}^{\prime}>1 / t_{j}^{\prime \prime}$ holds if and only if $v_{q 0}-v_{q j} t_{j}^{\prime}>0$ when $t_{j}^{\prime}>0$, and if and only if $v_{q 0}-v_{q j} t_{j}^{\prime}<0$ when $t_{j}^{\prime}<0$. These conditions reduce to the condition $v_{q j}<v_{q 0} / t_{j}^{\prime}$ on multiplying through by $1 / t_{j}^{\prime}$. However, $v_{k 0}-v_{k j} t_{j}^{\prime}=0$ from the definition of $t_{j}^{\prime}$, and by the normalization assumption this gives $1 / t_{j}^{\prime}=v_{k j}$. Consequently, we must show the restrictiveness condition is equivalent to $v_{q j}<v_{q 0} v_{k j}$. From the definition of $t_{j}^{\prime \prime}$ and the normalization assumption $\lambda_{k q}+\lambda_{q} v_{q 0}=1$ we have $1-\left(\lambda_{k q} v_{k j}+\lambda_{q} v_{q j}\right) t_{j}^{\prime \prime}=0$. Eliminating $\lambda_{k q}$ from this equation by the identify $\lambda_{k q}=1-\lambda_{q} v_{q 0}$, we obtain $1 / t_{j}^{\prime \prime}=v_{k j}+\lambda_{q}\left(v_{q j}-v_{q 0} v_{k j}\right)$. Thus $1 / t_{j}^{\prime \prime}>1 / t_{j}^{\prime \prime}$ if and only if $\lambda_{q}\left(v_{q j}-v_{q 0} v_{k j}\right)<0$. For $\lambda_{q}>0$, this is equivalent to $v_{q j}<v_{q 0} v_{k j}$, completing the proof.

The foregoing proof identifies algebraic relationships that are useful both in applying a sequential implementation strategy and in simplifying the proofs of subsequent results. These relationships may be summarized by

$$
\begin{aligned}
& 1 / t_{j}^{\prime}=v_{k j}, \quad 1 / t_{j}^{\prime \prime}=v_{k j}+\lambda_{q}\left(v_{q j}-v_{q 0} v_{k j}\right), \\
& 1 / t_{j}^{\prime}<1 / t_{j}^{\prime \prime} \quad \text { for } \lambda_{q}>0 \Leftrightarrow v_{q j}<v_{q 0} v_{k j},
\end{aligned}
$$

where $t_{j}^{\prime}$ and $t_{j}^{\prime \prime}$ are as defined previously. As the proof shows, (4) is an algebraic equivalent to the geometric assertions of the theorem. (This equivalent is a good deal less tidy without the normalization conventions.)

It should be noted that the assumption $\lambda_{q}>0$ of Theorem 5.1 is "superfluous" if $\lambda_{q}$ is legitimate, since there are always positive values of $\lambda_{q}$ satisfying $\lambda_{q} \geqslant 0$ and $\lambda_{q} v_{q 0} \leqslant 1$, and the selection of $\lambda_{q}$ to be 0 causes the half spaces $v_{k} \geqslant 0$ and $\lambda_{k q} v_{k}+\lambda_{q} v_{q} \geqslant 0$ to coincide, leaving nothing to be asserted.

In the "one at a time" manipulation of parameters by the sequential implementation strategy, it is desirable to know whether improved edge extensions may be obtained when the parameters $\lambda_{k h}$ of the hyperplane $v_{k}=0$ are held constant in Corollaries 4.4 and 4.5 , allowing only the parameters of the newly introduced hyperplanes to be varied. (This is of course relevant to any strategy in which candidates for new hyperplanes are screened by requiring that they afford improvement with the current values of the $\lambda_{k h}$ temporarily taken as given.) In this setting, Theorem 5.1 has the following consequences. 
Corollary 5.2. Assume the parameters $\lambda_{k h}$ are held constant and edge $j$ is blocked by the hyperplane $v_{k}=0$. Then for $\lambda^{\prime}, \lambda^{\prime \prime}>0$, the edge can become unblocked (i.e., allowed an improved extension) by both $v_{k}^{\prime}=0$ and $v_{k}^{\prime \prime}=0$ in Corollary 4.4 if and only if the intersection of edge $j$ with $v_{k}=0$ occurs for a positive value of $t_{j}$ and lies in the interior of $0 \leqslant u_{t} \leqslant 1$.

Proof. The supposition that $\lambda^{\prime}$ and $\lambda^{\prime \prime}$ are positive is imposed to avoid the degenerate situation in which $v_{k}^{\prime}$ or $v_{k}^{\prime \prime}$ is the same as $v_{k}$. Then, by Theorem 5.1, if the intersecting value of $t_{j}$ is negative, $v_{k}^{\prime}$ and $v_{k}^{\prime \prime}$ are both less restrictive than $v_{k}$ if and only if the point of intersection lies in the interior of $u_{t} \leqslant 0$ and $1-u_{t} \leqslant 0$, which is impossible. This leaves the case in which the intersecting value is positive, and the corollary follows at once.

This corollary shows that the use of $u_{t} \geqslant 0$ and $1-u_{t} \geqslant 0$ to modify (P) provides no help for negative edge extensions. For positive edge extensions, the fact that the point of intersection with the blocking hyperplane must lie in the interior of $0 \leqslant u_{t} \leqslant 1$ to permit the edge to become "unblocked" is highly useful.

Another consequence of Theorem 5.1 is the following.

Corollary 5.3. Under the assumptions of Corollary 5.2, the edge $j$ can become unblocked by $v_{k}^{\prime}=0$ of Corollary 4.5 if and only if the intersection of edge $j$ with $v_{k}=0$ satisfies $x_{i}<0$ for a positive intersection value of $t_{j}$ and satisfies $x_{i}>0$ for a negative intersection value of $t_{j}$.

Proof. Immediate by Theorem 5.1.

Corollary 5.3 has an interesting geometric interpretation. If the vertex of the LP cone is feasible for the LP problem, then it satisfies $x_{i} \geqslant 0$, and an edge extension (i.e., its endpoint) can satisfy $x_{i}<0$ only if it "bypasses" the hyperplane $x_{i}=0$. That is, a constraint hyperplane affords the chance of improving a positive edge extension only if this hyperplane has been encountered and left behind as the edge is extended.

Our next concern is to identify conditions that permit the hyperplane $\lambda_{k q} v_{k}+\lambda_{q} v_{q}=0$ to be rotated "out of the way" and thereby allow its associated half space to be discarded. Further, barring this possibility, we will want to know the least restrictive form of this half space relative to any given edge. 
The concept of a barrier hyperplane is relevant here. In particular, if the hyperplane $v_{k}=0$ is not a barrier for any edge, then it can be discarded immediately without bothering to replace it through the operations of Corollary 4.4 or 4.5 . The reason for this goes back to the assumption that the LP vertex lies in the interior of the half space $v_{k} \geqslant 0$, and thus if $v_{k j} \leqslant 0$ for all $j$ (which is equivalent to saying $v_{k}=0$ is not a barrier for any edge), then $v_{k}>0$ for all feasible MIP solutions (since, in fact, $v_{k} \geqslant v_{k 0}=1$ for $\left.t_{j} \geqslant 0, j \in N\right)$. Thus, our interest lies in examining situations in which $v_{k}=0$ is a barrier for some particular edge (or collection of edges). The first result that pertains to such situations is the following.

Theorem 5.4. Assume $v_{k}=0$ is a barrier for edge s and $v_{q}=0$ is not. Then the half space $\lambda_{k q} v_{k}+\lambda_{q} v_{q} \geqslant 0$ can be discarded provided the intersection of edge $s$ with $v_{k}=0$ lies in the interior of the half space $v_{q} \geqslant 0$ and $v_{k j} \leqslant \lambda_{q}^{*}\left(v_{q 0} v_{k j}-v_{q j}\right)$ for all $j \in N$, where $\lambda_{q}^{*}=$ $v_{k s} /\left(v_{q 0} v_{k s}-v_{q s}\right)$.

Proof. By (3), the condition $v_{k j} \leqslant \lambda_{q}\left(v_{q 0} v_{k j}-v_{q j}\right)$ is equivalent to $1 / t_{j}^{\prime \prime} \leqslant 0$, where by the definition of $t_{j}^{\prime \prime}$ and the normalization conventions we may write

$$
\lambda_{k q} v_{k}+\lambda_{q} v_{q}=1-\sum_{j \in N}\left(1 / t_{j}^{\prime \prime}\right) t_{j}
$$

Thus $\lambda_{k q} v_{k}+\lambda_{q} v_{q} \geqslant 0$ can be discarded provided the specified conditions can be achieved for a legitimate value of $\lambda_{q}$ - hence, in particular, if $\lambda_{q}=\lambda_{q}^{*}$ is legitimate. The fact that $v_{k}=0$ is a barrier for edge $s$ implies that it is intersected by the edge at a positive value of $t_{s}$ (since $v_{k 0}=1$ ). Then by the proof of Theorem 5.1, the assumption that this intersection lies in the interior of $v_{q} \geqslant 0$ is equivalent to $v_{q s}<v_{q 0} v_{k s}$, and hence $\lambda_{q}^{*}>0$ (since $v_{k s}>0$ ). It remains to show $\lambda_{q}^{*} v_{q 0} \leqslant 1$. The definition of $\lambda_{q}^{*}$ yields $1-\lambda_{q}^{*} v_{q 0}=-v_{q s} /\left(v_{q 0} v_{k s}-v_{q s}\right) \geqslant 0$, since $v_{q s} \leqslant 0$ under the assumption that $v_{q}=0$ is not a barrier for edge $s$. This completes the proof.

Geometrically, Theorem 5.4 specifies the conditions under which the hyperplane $\lambda_{k q} v_{k}+\lambda_{q} v_{q}=0$ can be rotated parallel to edge $s$ (for legitimate parameter values) and thereby become a "nonbarrier" for all edges.

Two principal "components" of the theorem, that $v_{q}=0$ is not a 
barrier for edge $s$ and that the intersection of edge $s$ with $v_{k}=0$ lies in the interior of $v_{q} \geqslant 0$, are crucially important. We make this explicit in the following observation.

Corollary 5.5. Let $B=\left\{j \in N: v_{k}=0\right.$ is a barrier for edge $\left.j\right\}$. Then there exists a legitimate value of $\lambda_{q}$ for which $\lambda_{k q} v_{k}+\lambda_{q} v_{q}=0$ is not a barrier for any edge (and hence can be discarded) if and only if the following hold: $v_{q}=0$ is not a barrier for each edge $j$ such that $j \in B$; the intersection of each edge $j, j \in B$, with $v_{k}=0$ lies in the interior of $v_{q} \geqslant 0$; $v_{k j} \leqslant \lambda_{q}^{*}\left(v_{q 0} v_{k j}-v_{q j}\right)$ for all edges $j$ for which $v_{q}=0$ is a barrier, where $\lambda_{q}^{*}$ is the maximum of $v_{k j} /\left(v_{q 0} v_{k j}-v_{q j}\right)$ over $j \in B$.

Proof. The assertion of the corollary follows from the proof of Theorem 5.4 by noting that $v_{k j}>0$ is compatible with $1 / t_{j}^{\prime \prime} \leqslant 0$, and $\lambda_{q}^{*}$ is legitimate, if and only if each of the stated assumptions hold. Further, the stipulated value of $\lambda_{q}^{*}$ of the corollary is the smallest value that assures $1 / t_{j}^{\prime \prime} \leqslant 0$ for all edges $j$ that are blocked by $v_{k}=0$, and hence if this value does not assure $1 / t_{j}^{\prime \prime} \leqslant 0$ for all remaining $j$ (as the inequalities of the corollary do), then no other value will assure this either.

Loosely speaking, the corollary says there must be some edge $s$ that satisfies the conditions of Theorem 5.4 or the hyperplane $\lambda_{k q} v_{k}+$ $\lambda_{q} v_{q}=0$ must remain a barrier for at least one edge and all legitimate values of $\lambda_{q}$.

It is useful to know circumstances under which the conditions of Theorem 5.4 may hold without having to check explicitly for these conditions. Specifically, the question arises as to the possibility of discarding $\lambda_{k q} v_{k}+\lambda_{q} v_{q} \geqslant 0$ without computing $\lambda_{q}^{*}$ and checking the associated inequalities. The following result deals with this situation.

Corollary 5.6. Assume $v_{q}=0$ is not a barrier for any edge, and for every edge $j$ for which $v_{k}=0$ is a barrier, the intersection of edge $j$ with $v_{k}=0$ is in the interior of $v_{q} \geqslant 0$. Then there exists a legitimate value of $\lambda_{q}$ such that $\lambda_{k q} v_{k}+\lambda_{q} v_{q} \geqslant 0$ can be discarded.

Proof. The corollary follows directly from Corollary 5.5, observing that the inequalities involving edges $j$ for which $v_{q}=0$ is a barrier do not apply, and hence $\lambda_{q}^{*}$ as specified in Corollary 5.5 must automatically be acceptable.

Corollary 5.6 is of particular relevance to "positive" coordinate 
systems, as in "bounding form" structures standardly employed to assure nonnegativity of nonbasic variables in creating matrix "normal forms" for integer programming, since for these it is always true that one of every pair of hyperplanes $u_{t}=0$ and $1-u_{t}=0$ is not a barrier for any edge.

Since it is not generally possible to rotate hyperplanes out of the way of all edges, it becomes of interest to know whether they may at least be rotated out of the way of "preferred" edges - e.g., edges that one would like to extend further. It is also desirable to know the best rotation available for a particular edge. The following corollary addresses this case.

Corollary 5.7. Assume $v_{k}=0$ is a barrier for the $j^{\text {th }}$ edge and the intersection of this edge with $v_{k}=0$ lies in the interior of $v_{q} \geqslant 0$. If $v_{q}=0$ is not a barrier for the $j^{\text {th }}$ edge, there is a legitimate value of $\lambda_{q}$ such that $\lambda_{k q} v_{k}+\lambda_{q} v_{q}=0$ is not intersected by this edge. If $v_{q}=0$ is a barrier for the jth edge, then the least restrictive form of the half space $\lambda_{k q} v_{k}+\lambda_{q} v_{q} \geqslant 0$ relative to this edge is obtained for $\lambda_{k q}=0$ (yiclding $v_{q} \geqslant 0$ in normalized form).

Proof. The first part of the corollary follows from Corollary 5.6. The second part requires the demonstration that if $v_{q j}>0$, then $\lambda_{k q} v_{k j}+$ $\lambda_{q} v_{q j}\left[\equiv v_{k j}+\lambda_{q}\left(v_{q j}-v_{q 0} v_{k j}\right)\right]$ is minimized for $\lambda_{k q}=0$, hence $\lambda_{q}=$ $1 / v_{q 0}$. As in the proof of Theorem 5.1, $v_{k j} v_{q 0}>v_{q j}$, and from $v_{k j}>0$ we have $v_{q 0}>v_{q j} / v_{k j}>0$. Thus $\lambda_{q}=1 / v_{q 0}$ is legitimate. Moreover, since $v_{q 0}>0, \lambda_{q}$ is required to satisfy $\lambda_{q} \leqslant 1 / v_{q 0}$ and $v_{k j}+$ $\lambda_{q}\left(v_{q j}-v_{q 0} v_{k j}\right)$ is minimized by making $\lambda_{q}$ as large as possible, since the quantity in parenthesis is negative.

The preceding results can be "compounded" in a sequential implementation strategy in order to permit a more effective use of Corollaries 4.4 and 4.5 in conjunction. Specifically, assume that $v_{q}$ of $\lambda_{k q} v_{k}+$ $\lambda_{q} v_{q}$ corresponds to $u_{t}$ or $1-u_{t}$ in Corollary 4.4 , and values of the parameters are sought that will achieve the best combined set of rotations on the subsequent application of Corollary 4.5. Then by Theorem 5.1 (and Corollary 5.3), the parameters should be selected so that $-x_{i}=0$ is intersected by a given edge $j$ "before" the edge intersects $\lambda_{k q} v_{k}+$ $\lambda_{q} v_{q}=0$. For negative edge extensions, the appropriate sequence of intersections is reversed. However, the more nearly that the hyperplane $\lambda_{k q} v_{k}+\lambda_{q} v_{q}=0$ can be brought into a position so that it satisfies the 
conditions of Theorem 5.4 relative to $-x_{i}=0$, then the better the resulting rotation will be. This requires the manipulation of two parameters. However, since a "best" value of the second parameter is always known (e.g., by Corollary 5.5) for each value of the first, the procedure simplifies to a search over one parameter only.

In general, the simplest type of sequential implementation strategy (i.e., requiring the least calculation), favors a rotation as long as it allows at least one edge extension to be improved without worsening any other edge extension.

The algebraic relations underlying such an approach are completely straightforward and may be summarized as follows.

Remark. Let $t_{j}^{*}, j \in N$, be determined relative to (P) and suppose $v_{k}=0$ blocks at least one of the edges of the LP cone. Upon replacing $v_{k} \geqslant 0$ with $\lambda_{k q} v_{k}+\lambda_{q} v_{q} \geqslant 0$ the greatest improvement in the extensions of the blocked edges that does not worsen the extensions of any other edges is given by assigning $\lambda_{q}$ the largest value satisfying $\lambda_{q} v_{q 0} \leqslant 1$ and

$$
\lambda_{q} \leqslant\left(v_{k j}-1 / t_{j}^{*}\right) /\left(v_{q 0} v_{k j}-v_{q j}\right)
$$

for $j \in N: v_{q 0} v_{k j}>v_{q j}{ }^{9}$

Proof. The inequalities other than $\lambda_{q} v_{q 0} \leqslant 1$ are the requirements $1 / t_{j}^{\prime \prime} \leqslant 1 / t_{j}^{*}, j \in N$, expressed in terms of the LP basis representation, utilizing the identities developed in the proof of Theorem 5.1.

\subsection{Linear programming}

The formulation of an auxiliary linear programming problem to determine optimal parameter values $\lambda_{p h}$ for the polyhedron (P) is easily derived (for $(\mathrm{P})$ in the form $\left(\mathrm{P}_{\lambda}\right)$ ), given a prior specification of the variables $v_{h}$, by means of which $(\mathrm{P})$ is parameterized. This is accomplished by imposing the normalization $v_{p 0}=1, p \in P$ (to insure the LP vertex is in the interior of (P)), and stipulating $\delta_{j} \geqslant v_{p j}, p \in P, j \in N$, where $\delta_{j}$ represents the coefficient of $t_{j}$ in $(1)-$ i.e., $\delta_{j}^{*}=1 / t_{j}^{*}$, for a given value $\delta_{j}^{*}$ of $\delta_{j}$. (The inequality $\delta_{j} \geqslant v_{p j}$ is equivalent to $v_{p 0} \geqslant$ $v_{p j} t_{j}$ for $v_{p 0}=1$ and $\delta_{j}=1 / t_{j}$.) Since stronger cuts are obtained for smaller values of $\delta_{j}$, the objective for the auxiliary linear program can be to minimize the sum of the $\delta_{j}$, or more generally

9 Of course, it may be that no improvement is possible by the remark once a "local optimum" has been obtained, in which case $\lambda_{q}=0$. (Edges blocked by more than one hyperplane will require all blocking hyperplanes to be replaced.) 


$$
\operatorname{minimize} \sum_{j \in N} d_{j} \delta_{j}
$$

where the coefficients $d_{j}$ are selected to be positive - for example, with magnitudes of the coefficients $x_{0 j}$ in the updated objective function for the MIP problem. From $\left(\mathrm{P}_{\lambda}\right)$ the requirements $v_{p 0}=1$ and $\delta_{j} \geqslant v_{p j}$ become

$$
\begin{aligned}
& \sum_{h \in H_{p}} \lambda_{p h} v_{h 0}=1, \quad p \in P, \\
& \delta_{j} \geqslant \sum_{h \in H_{p}} \lambda_{p h} v_{h j}, \quad p \in P, \quad j \in N,
\end{aligned}
$$

where $\lambda_{p h} \geqslant 0, h \in H_{p}, p \in P$. The variables $\delta_{j}$ are unrestricted. Some of the parameters $\lambda_{p h}$ may also be unrestricted in accordance with the observation following Corollary 4.5 . The variables $v_{h}$ consist of the "building block" variables, hence in the MIP setting are the variables $u_{t}, 1-u_{t}$, and $-x_{i}$, from Corollaries 4.4 and 4.5 . The particular variables to be used may be identified in advance through a heuristic means such as the sequential implementation approach. Alternatively, both variables and "starting solutions" may be obtained by reference to earlier solution attempts involving problems from a similar class.

In any of these approaches, upon starting from a polyhedron $(\mathrm{P})$ that contains the LP vertex in its interior, additional variables $v_{h}$ may be introduced using row and column generating procedures for the primal simplex method as a means of implementing Corollaries 4.4 and 4.5 .

If one permits new variables to be introduced (either singly or in blocks) as long as it is profitable to do so, then the optimum solution to the linear program must yield a support of the convex hull $F^{*}$ of $F$ as a result of Corollary 4.3. (Otherwise, it would be possible to decrease some $\delta_{j}$ and obtain a better LP solution.) Clearly, too, every support (and hence every fact) indicated in Corollary 4.3 must be an optimal LP solution for some choice of positive coefficients in the objective function. In fact, each optimum extreme point solution of the LP problem must yield a fact of $F^{*}$, since, if $\delta t=1$ were not a facet then $\delta$ would have a degree of freedom to move along a line segment for finite distances in both directions, still yielding a support (and hence a feasible LP solution), contrary to the assumption that $\delta$ is an extreme point. 10 
To implement the approach of generating new variables as needed, the introduction of a new variable $-x_{i}$ by Corollary 4.5 is accomplished in a straightforward fashion. Specifically, this involves the creation of the new parameter $\lambda^{\prime}$, which appears in the constraints associated with $v_{k}$ in the auxiliary linear program:

$$
\begin{aligned}
& \sum_{h \in H_{k}} \lambda_{k h} v_{h 0}+\lambda^{\prime}\left(-x_{i 0}\right)=1, \\
& \delta_{j} \geqslant \sum_{h \in H_{k}} \lambda_{k h} v_{h j}+\lambda^{\prime}\left(-x_{i j}\right), \quad j \in N .
\end{aligned}
$$

Thus, the customary "pricing-out" procedure of linear programming can be used to determine whether it is profitable to introduce $-x_{i}$ (hence $\lambda^{\prime}$ ) in the indicated constraints (and thus change the identity of a particular $v_{k}, k \in P$ ). Theorem 5.1 - and more specifically, Corollary 5.3 - may be used to limit the range of variables $-x_{i}, i \in M$, that need to be examined. From these results, unless the endpoint of an edge blocked by $v_{k}=0$ (in the current solution to the auxiliary linear program) satisfies $-x_{i}>0$, or can be made to satisfy $-x_{i}>0$ for other feasible parameter values, then $-x_{i}$ (i.e., $\lambda^{\prime}$ ) cannot be introduced profitably. ( $-x_{i}>0$ is replaced by $-x_{i}<0$ for negative edge extensions.) In particular, then, if edge $j$ is blocked by $v_{k}=0$, and $\delta_{j}^{*}$ is the current value of $\delta_{j}$, then $-x_{i}$ need not be priced-out unless $\delta_{j}^{*} x_{i 0}-x_{i j}$ is negative or can be made negative for a relatively small change in $\delta_{j}^{*}$.

To introduce new variables $u_{t}$ and $1-u_{t}$ by Corollary 4.4 requires more effort. Here the constraints of the auxiliary linear program associated with $v_{k}$ become

$$
\begin{aligned}
& \sum_{h \in H_{k}} \lambda_{k h}^{\prime} v_{h 0}+\lambda^{\prime} u_{t 0}=1, \\
& \sum_{h \in H_{k}} \lambda_{k h}^{\prime \prime} v_{h 0}+\lambda^{\prime \prime}\left(1-u_{t 0}\right)=1 ; \\
& \delta_{j} \geqslant \sum_{h \in H_{k}} \lambda_{k h}^{\prime} v_{h j}+\lambda^{\prime} u_{t j},
\end{aligned}
$$

10

This correspondence between extreme point solutions and facets is related to similar correspondences noted by Gomory [18], Balas [4] and Johnson [23]. However, in the present setting, $F^{*}$ may not have $n$-dimensional facets, or may otherwise lack facets for particular choices of the coefficients $d_{j}$ that prevent the objective function from being unbounded. This causes no difficulty if the $\delta_{j}$ are bounded from below. 


$$
\delta_{j} \geqslant \sum_{h \in H_{k}} \lambda_{k h}^{\prime \prime} v_{h j}+\lambda^{\prime \prime}\left(-u_{t j}\right), \quad j \in N .
$$

Note that the original parameters $\lambda_{k h}, h \in H_{k}$, can be taken to correspond to either the parameters $\lambda_{k h}^{\prime}$ or $\lambda_{k h}^{\prime \prime}$. This implies that if either $u_{t}$ or $1-u_{t}$ (i.e., $\lambda^{\prime}$ or $\lambda^{\prime \prime}$ ) prices-out unprofitably in the auxiliary linear program (regardless of whether the other prices-out profitably, and without bothering to create additional constraints or parameters) then there is no use attempting to introduce these variables. This fact can result in substantial savings of time and effort. On the other hand, if both $u_{t}$ and $1-u_{t}$ price-out profitably, then row and column generating procedures are required to introduce these variables and their associated constraints. Ways of carrying out this process efficiently are extremely important, and alternatives merit careful study. The results of Section 5.1 that give sufficient conditions for discarding newly created half spaces can be helpful in this regard, since they allow the application of Corollary 4.4 to be restricted to only one of the two variables $u_{t}$ and $1-u_{t}$, thereby effectively reducing the work of implementing Corollary 4.4 to the level involved in implementing Corollary 4.5 .

Theorem 5.1 can also be used to screen variables $u_{t}$ and $1-u_{t}$ to be priced-out in the first place (just as for the variables $-x_{i}$ ). Specifically, by Corollary 5.2, if edge $j$ is blocked by $v_{k}=0$, the introduction of $u_{t}$ and $1-u_{t}$ can be useful only if the endpoint of this edge lies in the interior of $0 \leqslant u_{t} \leqslant 1$, or can be made to do so for other feasible parameter values. That is, $\delta_{j}^{*}$ must be positive and the inequality $\delta_{j}\left(u_{t 0}-1\right)<u_{t j}<\delta_{j} u_{t 0}$ must hold for $\delta_{j}$ in the vicinity of $\delta_{j}^{*}$. (As in the corresponding implementation of Corollary 4.5, the size of this "vicinity" depends on how stringent one desires the screening device to be.)

It is important to bear in mind that these updating operations do not destroy the primal feasibility of the auxiliary linear program, thus permitting termination at any selected stage.

\section{Applications in branch and bound}

The results of Sections 2 and 3 are especially suitable for implementation in the context of branch and bound. It has often been noted, for example, that cutting inequalities can be used to obtain improved bounds in a branch and bound approach. However, the preceding results 
give a new way to take advantage of "branching inequalities," since these inequalities may be conceived to have the form $x_{i} \geqslant 0$, just as the original problem constraints. Corollary 4.5 is therefore immediately applicable, and it is not necessary to iterate to a new LP optimum before exploiting its consequences. Moreover, since the LP vertex will satisfy $x_{i}<0$ for a branching inequality, it follows that positive extensions of many of the edges will also satisfy $x_{i}<0$, and hence by Corollary 5.2 , the constraint $x_{i} \geqslant 0$ can automatically be used to improve the extensions of these edges.

Improved applications of branch and bound in this setting may occur by branching on variables $u_{t}$ and $1-u_{t}$ created by easily identifiable integer combinations of the original integer variables that allow deeper edge extensions relative to the current LP basis than the original variables themselves. However, somewhat more general procedures can be employed by reference to polyhedra generated by the approaches of the preceding sections. Specifically, if there is a hyperplane $v_{k}=0$ of (P) which by itself would provide a strong cut $\left(v_{k} \leqslant 0\right)$ in the role of $(\mathrm{P})$, and if the remaining polyhedron $v_{p} \geqslant 0, p \in P-\{k\}$ also provides a strong cut, then it is possible to "branch on $v_{k}$ " by imposing $v_{k} \leqslant 0$ as one alternative, and by imposing the cut (1) from the polyhedron $v_{p} \geqslant 0, p \in P-\{k\}$, together with the negation of $v_{k} \leqslant 0$, as the other alternative.

This is an instance of the following rule. If $P^{\prime}$ and $P^{\prime \prime}$ are subsets of $P$, with $P^{\prime} \cup P^{\prime \prime}=P$, then the branching may consist of the disjunction

$$
\sum_{j \in N}\left(1 / t_{j}^{\prime}\right) t_{j} \geqslant 1
$$

or

$$
\begin{aligned}
& \sum_{j \in N}\left(1 / t_{j}^{\prime \prime}\right) t_{j} \geqslant 1, \\
& \sum_{j \in N}\left(1 / t_{j}^{\prime}\right) t_{j} \leqslant 1-\epsilon,
\end{aligned}
$$

where $t_{j}^{\prime}$ and $t_{j}^{\prime \prime}$ are the values of $t_{j}^{*}$ for the polyhedra $v_{p} \geqslant 0, p \in P^{\prime}$ and $v_{p} \geqslant 0, p \in P^{\prime \prime}$, respectively. The number $\epsilon$ can safely be 0 , but may be positive when the slack variable for the associated inequality is identified as a scaled integer variable. Similar observations give rise to multiple branchings.

The potential effectiveness of such possibilities should not be under- 
estimated, since customary branch and bound approaches are special cases of them. The latitude to generate polyhedra that provide branching inequalities with deeper edge extensions suggests the possible desirability of using branching strategies based on such considerations in branch and bound algorithms.

\section{References}

[1] E. Balas, "The intersection cut - A new cutting plane for integer programming", Operations Research, 19 (1970) 19-39.

[2] E. Balas, "Integer programming and convex analysis: Intersection cuts from outer polars", Mathematical Programming 2 (3) (1972) 330-382.

[3] E. Balas, "On the use of intersection cuts in branch and bound", paper presented at the $8^{\text {th }}$ International Symposium on Mathematical Programming, Stanford, August 1973.

[4] E. Balas, "Disjunctive programming: Properties of the convex hull of feasible points", Management Science Research Rept. No. 348, Camegie-Mellon University (July 1974).

[5] C.-A. Burdet, "Enumerative cuts: I", Operations Research 21 (1) (1973) 61-89.

16] C.-A. Burdet, "Polaroids: a new tool in non-convex and in integer programming", Naval Research Logistics Quarterly (1973) $13 \cdots 24$.

17] C. Burdet, "Convex and polaroid extensions", Rept. \#73-21, Ottawa (October 1973).

181 C. Burdet, "On the algebra and geometry of integer cuts", M.S. Rept. 291, CarnegicMellon University (1972), revised and extended in Rept. \#74-8, Ottawa (April 1974).

19] R.J. Dakin, "A tree search algorithm for mixed integer programming problems", Computer Journal 8 (3) (1965) 250-255.

[10] [: Glover, "Convexity cuts and cut search", Operations Research 21 (1) (1973) $123 \cdots 134$.

[11] F. Glover, "Convexity cuts for multiple choice problems", Discrete Mathematics 6 (1973) $221-234$.

[12] F. Glover, "Polyhedral convexity cuts and negative edge extensions", MSRS 73-6, University of Colorado (May 1973).

[13] F. Glover and D. Klingman, "The generalized lattice point problem", Operations Research 21 (1) (1973) $141-156$.

[14] F. Glover, D. Klingman and J. Stutz, "The disjunctive facet problem: Formulation and solution techniques", Management Science 22 (3) (1974) 582--601.

[15] F. Glover and D. Sommer, "Inequalities for mixed integer prograns with structure", MSRS 73-2, University of Colorado (lebruary 1973).

[16] F. Glover, "Polyhedral annexation and the convex hull of feasible solutions to mixed integer programs", MSRS 74-3, University of Colorado (March 1974).

[17] R.E. Gomory, "An algorithm for the mixed integer problems", Research Memorandum RM-2597, Rand Corporation, Santa Monica (1960).

[18] R.E. Gomory, "Some polyhedra related to combinatorial problems", Journal of Linear Algebra and Applications 2 (4) (1969) 451-558.

[19] R.E. Gomory and L.L. Johnson, "Some continuous functions related to convex polyhedra", Mathematical Programming 3 (1) (1972) 23-85.

[20] R.G. Jeroslow, "The principles of cutting-plane theory: Part I" (with an addendum), GSIA, Carnegie-Mellon University (February 1974).

[21] R.G. Jeroslow, "Relaxations of integer programs", Management Science Research Rept. No. 347, Carncgie-Mellon University (July 1974). 
188 F. Glover/Polyhedral annexation in mixed integer and combinatorial programming

[22] E. Johnson, "On the group problem for mixed integer programming", Mathematical Programming Study 2 (1974) 137-179.

[23] E.L. Johnson, "Integer programs with continuous variables", Operations Research Institute, University of Bonn (July 1974).

[24] G. Owen, "Cutting planes for programs with disjunctive constraints", Journal of Optimization Theory and Applications 11 (1) (1973) 49-55.

[25] H. Tui, "Concave programming under linear constraints", Doklady Akademii Nauk SSSR (1964) [in Russian; English transl. Soviet Mathematics (1964) 1437-1440].

[26] R.D. Young, "Hypercylindrically deduced cuts in zero-one integer programming", Operations Research 19 (1971) 1393-1405. 